\title{
THE MATERNAL-FETAL MEDICINE: AN UPDATE
}

\section{Vincenzo Berghella, ${ }^{1}$ Alessandro Ghidini, ${ }^{2}$ Giancarlo Mari, ${ }^{3}$ Mariarosaria Di Tommaso, ${ }^{4}$ Silvia Vannuccini, ${ }^{5}$ Filiberto Maria Severi, ${ }^{5}$ Felice Petraglia ${ }^{5}$}

\begin{abstract}
${ }^{1}$ Division of Maternal-Fetal Medicine, Department of Obstetrics and Gynecology, Jefferson Medical College of Thomas Jefferson University, Philadelphia, Pennsylvania, USA; ${ }^{2}$ Department of Obstetrics and Gynecology, Georgetown University Hospital, Washington DC, USA; ${ }^{3}$ Department of Obstetrics and Gynecology, University of Tennessee Health Science Center, College Of Medicine, Memphis, USA;

${ }^{4}$ Department of Child and Woman's Health, University of Florence, Careggi University Hospital, Florence, Italy; ${ }^{5}$ Department of Molecular and Developmental Medicine, University of Siena, Siena, Italy
\end{abstract}

\begin{abstract}
The development of Maternal-Fetal Medicine is contributing to an improvement of maternal well-being and of neonatal health, introducing a number of new and useful technologies. Advances in genomics in the field of prenatal screening and diagnosis allowed the discovery of fragments of cell-free fetal DNA in the maternal circulation and the use of chromosomal microarrays, which can test for microdeletions and microduplications in addition to aneuploidies.

Color Doppler applications during pregnancy are expanding exponentially and Doppler flow velocity waveforms indices have provided important information from maternal, placental and fetal circulation with clinical implications. Ultrasound monitoring of fetal growth represents a fundamental tool to evaluate fetal wellbeing and several methods have been developed to improve fetal weight estimation accuracy.

The combination of new biophysical and biochemical markers is enriching Maternal-Fetal Medicine and more research will allow to improve pregnancy outcome.
\end{abstract}

Key words: prenatal diagnosis, Doppler analysis, fetal anemia, fetal growth, preterm birth.

\section{INTRODUCTION}

Perinatal medicine has grown very fast in the last two decades, introducing a number of technologies useful for monitoring maternal and fetal health, thus it has been renamed as Maternal-Fetal Medicine. The present review aims to follow up some of the most update topics: prenatal diagnosis, fetal circulation, fetal anemia, fetal growth and cervical insufficiency.

\section{PRENATAL SCREENING AND DIAGNOSIS}

Traditionally, screening for genetic conditions has focused on the most common chromosome abnormalities, which are Trisomy 21, Trisomy 13 and Trisomy 1 [1]. Indeed, these 3 conditions combined account for $71 \%$ of all chromosome anomalies. In addition, screening for certain single gene disorders (such as Thalassemia or sickle cell disease) can be offered depending on ethnicity. Women identified as having increased risk of an affected fetus based on family his- tory or screening test result can then be offered invasive prenatal diagnosis. Advances in genomics has rapidly revolutionized both the field of prenatal screening and diagnosis.

\section{Genomics and prenatal screening for fetal aneuploidies}

Over the 1980s and 1990s, several markers of Down syndrome (detectable in maternal serum or at ultrasonography during the second trimester) were introduced to improve the detection rate of the most common trisomies[2]. Combining such markers allows the detection of up to $90 \%$ of trisomy 21 fetuses $[2,3]$ however at the expense of a false positive rates of at least $5 \%$, with rates increasing proportionately to maternal age.

During the 1990s, the impetus was on the identification of serum and sonographic markers in the first trimester, under the premise that early prenatal screening allows earlier prenatal diagnosis. Several large trials confirmed the ability of first trimester serum markers (e.g. Pregnancy-Associated plasma protein-A, or PAPP-A, and hCG), used in combination with 1st trimester sonographic markers (typically nuchal translucency) or with second trimester serum markers 
in a sequential fashion, to detect close to $85 \%$ of pregnancies affected by fetal Down syndrome.

The discovery that fragments of cell-free (cf) fetal DNA are present in the maternal circulation has opened the door to genomics-based prenatal screening [4]. cf-Fetal DNA fragments are smaller than cf-maternal DNA fragments, they account for about $10 \%$ of total cf-DNA in maternal blood, they are present from as early as 32 days after conception until delivery (being rapidly cleared from maternal circulation at delivery), and can be identified with a variety of methods (such as allele specific methods or methylation-specific methods). The entire fetal genome is present in fragments in the maternal plasma; the chromosome of origin can be identified via sequencing of as little as 36 nucleotides. The total number of cf-fetal DNA fragments of any one chromosome is proportional to the size of that chromosome and it is consistent from sample to sample, and from patient to patient. The fragments belonging to a specific chromosome can be quantified, e.g. using massively parallel sequencing, and the ratio of sequence reads mapping to a specific chromosome (e.g. chromosome 21 ) in a patient plasma can be compared with the expected values (e.g. from known independent samples). Identification of an excess amount of cf-DNA fragments from chromosome 21 compared with the expected increases the likelihood of fetal trisomy 21 . Similar calculations can be made for fragments from chromosome 18 and 13; fetal sex chromosome aneuploidies can also be identified with a high degree of accuracy based on DNA fragments from the sex chromosomes.

The main benefits of the cf-DNA test are in the very high detection rate and negligible false positive rates, thus resulting in reduced exposure to invasive prenatal procedures with associated risks. Table 1 displays the accuracy of cf-DNA testing according to the published data from prospective cohort studies on singleton pregnancies [5-7]. The test is typically performed after 10 weeks to allow reliable presence of cf-fetal DNA in the sample. Leading societies, including the International Society of Prenatal Diagnosis (ISPD), American Col- lege of Obstetrics and Gynecology (ACOG), Society of maternal Fetal Medicine (SMFM), and the National Society of Genetic Counselors (NSGC) currently recommend that the test be offered only to women with high risk pregnancies. Studies are being published and are underway in low risk populations. The challenge is currently to translate this technology into practice that is accessible to all pregnant women, in an ethical way that preserves informed parental choice, while not increasing overall costs to the health care systems.

Positive results at cf-DNA testing still require invasive testing because the positive predictive value changes with the prevalence of the condition. On the positive side, early and accurate diagnosis of trisomy 21 opens the door for prenatal treatment of the condition $[8,9]$.Other advantages of the new cf-DNA test include its independence from gestational age at screening and not being affected by maternal conditions, such as diabetes or smoking. Moreover the test shows a definite and advantageous cost-benefit ratio in high risk women. However, the amount of cf-DNA is affected by maternal obesity, apparently due to the large amount of cf-DNA originated by the adipose tissue. Other limitations of the test include the lack of oversight of the validity of the test. All the companies providing cf-DNA testing are commercial, thus under strong competitive pressure to expand indications before data on accuracy are available. For example, the original data on the accuracy of the test were derived from singleton pregnancies (Table 1). Recently twin gestations have been added with claims of high accuracy, however no data have been published.

cf-DNA testing for the most common aneuploidies opens the door to more in depth sequencing of cf-fetal DNA in maternal blood, with potential for broader detection of genetic abnormalities. Non-invasive fetal whole genome sequencing has already been reported in a recent proof-of-concept study [10]. Whole genome deep sequencing has opened the door to detection of microdeletions and microduplications with sizes ranging from $3 \mathrm{Mb}-40 \mathrm{Mb}$ [11], allowing detection of DiGeorge syndrome (22q11), Cri-du-chat syndrome (5p),

Table 1. Performance of cell-free DNA tests (using massively parallel sequencing) from prospective studies.

\begin{tabular}{lcccc}
\hline Test & \# patients enrolled & Ineligible samples* & Detection rate & Specificity \\
\hline Palomaki et al., 2011 & 1683 & $7.3 \%$ & $\begin{array}{c}98.6 \% \\
(96.4-99.6)\end{array}$ & $\begin{array}{c}99.8 \% \\
(99.5-99.9)\end{array}$ \\
\hline Bianchi et al., 2012 & 2882 & & $100 \%$ & $100 \%$ \\
& & $8.2 \%$ & $(95.9-100)$ & $(99.1-100)$ \\
\hline Norton et al., 2012 & 3569 & & $100 \%$ & $99.9 \%$ \\
& & $7.8 \%$ & $(94.6-100)$ & $(98.8-100)$ \\
\hline
\end{tabular}

*Including samples without adequate amount of cell-free fetal DNA and failed quality control. 
Angelman syndrome 8 Prader-Willis syndrome (15q), lp36 deletion syndrome (lp), as well as of other trisomies, e.g. Trisomy 22. For this enhanced sequencing, sensitivities of $94 \%$ (95\% CI 71-99\%) and false positive rate of $1 \%$ (95\% CI $1-5 \%$ ) have been described. Companies providing targeted cf-DNA testing for trisomy 13,18 and 21 cannot obviously perform deep sequencing for genome segments outside of such chromosomes. If non-invasive determination of the entire fetal genome becomes clinically available, there will be a significant number of ethical issues to be addressed as well as counseling challenges.

\section{Genomics and prenatal screening for single gene disorders}

Historically screening for single gene disorders has been offered based on maternal ethnicity. In addition family history of unexplained mental retardation, developmental disabilities or autism is an indication for offering testing for Fragile $\mathrm{X}$. Consanguinity is also known to increase the risk for single gene disorders, though no testing could be offered until recently in the absence of affected individuals.

Universal screening for over 100 single gene conditions is now available at limited cost, and can be done by saliva or blood sample from both parents. It has been shown that about $24 \%$ of individuals tested are found to be carriers for one of the tested conditions, with $<1 \%$ of couples being carriers of the same condition [12]. The most common carrier frequencies for single-gene disorders include spinal muscular atrophy, familial Mediterranean fever, cystic fibrosis, GJB2 connexin gene mutation (the most common nonsyndromic cause of congenital deafness), Smith Lemliopitz syndrome, sickle cell and thalassemia anemias, Tay Sachs and Gaucher disease. Detection of carrier status for the same condition in both parents allows planning future childbearing or accessing prenatal diagnostic tests during pregnancy.

\section{Prenatal diagnosis in the age of genomics}

Fetal cells obtained at chorionic villus sampling or amniocentesis can be used for karyotyping via traditional cytogenetic testing. Genomics now allows the use of chromosomal microarrays (e.g. based on Comparative Genomic Hybridization, $\mathrm{CGH}$, or Single $\mathrm{Nu}$ cleotide Polymorphisms), which can test for microdeletions and microduplications in addition to aneuploidies. The resolution of microarrays depends on the size of the backbone used: for example, in CGH, although the technology has the potential for resolution below $1 \mathrm{~kb}$, in practice the backbone used are $>100 \mathrm{~kb}$ in size. In comparison, high-resolution karyotypes allow a resolution of 2000-3000 kb (at the 8001000 band stage).

A large study has evaluated the accuracy of CGH compared with karyotyping in prenatal diagnosis [13]. Samples were obtained from chorionic villus sampling or amniocentesis in 4406 women with standard indications for prenatal diagnosis. Microarray was successful in $\mathbf{9 8 . 8 \%}$ of fetal samples, and in $\mathbf{8 7 . 9 \%}$ without tissue culture necessary. Microdeletions and microduplications were classified as benign, pathogenic, or of uncertain clinical significance. The study showed that all aneuploidies detected at traditional karyotyping were also detected at CGH, with the exception of balanced chromosome translocations, inversions and triploidy. However CGH was able to detect microdeletions or microduplications of pathogenic or potential for clinical significance in $2.5 \%$ of the cases with normal karyotype (among samples drawn in the absence of ultrasonographic fetal anomalies the rate was $1.6 \%$ ).

Advantages of microarrays include the higher resolution, the avoidance of culturing amniocytes and chorionic villi in the majority of cases, the lack of need for dividing cells (making the technique appealing in cases of fetal death), and automation (allowing better quality control and faster turnaround times). The main disadvantages of CGH are the inability to detect balanced translocations, inversions or triploidy. Cost is also an issue at present, as the detection of de novo microdeletions and microduplications is of unknown clinical significance. The latter issue is being addressed by the establishment of large databases of both healthy and affected individuals, with post-natal follow-up (indeed, databases derived from post-natal populations cannot be extrapolated to fetal populations). Currently, microarrays are recommended in the presence of: 1) fetuses with structural anomalies and normal karyotype (approximately $5 \%-10 \%$ of them will be found to have microdeletions or microduplications of clinical significance); in stillbirth: a large study has found that use of CGH compared with traditional karyotyping significantly increases the yield $(87 \%$ vs $70 \%, \mathrm{p}<0.01)$ and the detection of aneuploidy or pathogenic genomic imbalances $(8 \%$ vs $6 \%, \mathrm{p}<0.01)$, independently from the presence of anomalies [14]. It is expected that microarrays will soon replace traditional karyotyping (few karyotyped cells per sample will still be required in addition to $\mathrm{CGH}$ to detect triploidies and balanced translocations). Because of the ability to detect a variety of microdeletions and microduplications, some with a range of clinical presentation, microarrays can be a counseling challenge.

\section{FETAL CIRCULATION}

Fetal circulation was first described by William Harvey in 1628 and later attempts to study cardiovascular function in living fetuses were first reported by Cohnstein and Zuntz in 1884 that measured umbilical arterial pressure in exposed lamb fetuses. Traditionally fetal circulation was studied in sheep and goats following fetal delivery, but with continuing placental circu- 
lation, obtaining some of the most important data on fetal physiology and fetal circulation $[15,16]$. Later Dawes and his coworkers, using animal preparation with catheterizations of maternal and fetal vessels, were able to estimate the proportion of blood distributed through the vascular shunts of the foramen ovale and ductus arterious. A major advantage of the use of electronic flow meters for recording flows was a continuous observation over a period of hours [17]. In all these studies regarding fetal blood flow, the major limitations were due to the exteriorization of the fetus and the surgical manipulation, which prevented studying the circulation of the undisturbed fetus in utero.

In an effort to study the fetus in its intrauterine environment, Rudolph et al. introduced a sheep model chronically instrumented that allowed to study fetal circulation a few days after recovering from surgery by injection of nuclide-labeled microspheres, as an indicator to measure fractional distribution of blood flow to various organs [18]. The advent of non-invasive, ultrasonography techniques in the late 1970s, opened the exciting possibility of studying fetal circulation also in humans. First of all, Fitzgerald and Drumm combined two ultrasound techniques, real time imaging and pulsed Doppler system, to develop a safe, non-invasive, transcutaneous method of observing the circulation in the umbilical arteries and vein of a human fetus [19].

\section{Clinical use}

The science of ultrasound has exploded since the 1980s and obstetrics changed forever. Two are the applications of Doppler in the study of human fetal circulation.

1. Quantitative evaluation of fetal blood flow volume by Doppler velocimetry utilized the calculation of circulating volume measurements in the target organ by measuring vessel diameters and by detecting the absolute velocity in fetal vessels [20]. Velocity and volume measurements were performed in large vessels such as descending aorta, pulmonary artery, the umbilical vein or the intracardiac chambers. Since the quantitative doppler measurements of blood flow were affected by several errors, the main utility of Doppler velocimetry has shifted to the analysis of blood flow shape.

2. Qualitative assessment of blood flow in the human fetus is based on three indices derived from the qualitative analysis of Doppler flow velocity waveforms: pulsatility index (PI), A/B ratio, and resistance index (RI), which are the most commonly used parameters. These indices detected in the umbilical artery are considered indicators of placental vascular resistance [21]. From a technological point of view, the introduction of Color Doppler following that, which converts Doppler sounds into colors and thus produces a picture of blood flow, has allowed easier detection of abnormal flow patterns and determination of the sampling point.
Doppler applications in pregnancy are expanding exponentially and Doppler flow velocity waveforms indices provide important information from maternal, placental and fetal circulation with clinical implications both for the mother and the fetus. Placental development can be documented by assessing uterine and umbilical arteries. Uterine arteries flow velocity shows the maternal vascular effects of the invading placenta and reflects downstream placental vascular resistance, strongly correlated with intrauterine growth restriction and the multisystem effects of placental deficiency. Abnormalities are progressive, with reduction, loss, and finally a reversal of diastolic flow [22].

Umbilical artery flow velocity waveforms provide an estimate of villous vascular occlusion in the fetal compartment of the placenta. The studies of both these vessels, uterine and umbilical arteries are useful for the prediction of hypertensive maternal disorders, placental dysfunction and fetal growth failure. When umbilical arteries become abnormal, the differentiation of fetal status requires Doppler information from systemic vessels.

Middle cerebral artery changes begin when the redistribution of cardiac output happens and reflects increasing placental resistance, demonstrating 'brain sparing' when cerebrovascular dilation occurs. Middle cerebral artery Doppler is important not only in studying fetal responses to abnormal oxygenation but also to detect fetal anemia [23].

With the progression of a compromised intrauterine growth, precordial venous Doppler of fetal cardiac function changes as the oxygenation status declines. Because of this, the ductusvenosus Doppler has become useful today in the management of conditions that put the fetus at risk for cardiovascular deterioration. Doppler venous information, combined with modified biophysical profile, is used to determine the need for and timing of delivery. Besides fetal growth restriction, venous Doppler gives information of cardiac effects of other conditions such as twin-twin transfusion syndrome and fetal arrhythmia [24].

\section{FETAL ANEMIA}

Fetal anemia is defined as a hemoglobin value below two standard deviations from the mean. Fetal anemia is categorized as mild, moderate and severe. Severe anemia may cause hydrops and fetal demise [25].

\section{Red cell alloimmunization}

Red cell alloimmunization remains one of the most common causes of fetal anemia in the world. The introduction of $\mathrm{Rh}(\mathrm{D})$ immune globulin in 1968 has almost eliminated fetal anemia due to $\mathrm{Rh}$ alloimmunization in North America. However, red cell alloimmunization due to other antigens (Kell, Kidd, 
Duffy, etc.) is still a problem, and it will continue to be present until a new policy for blood transfusion is developed. In fact, in the United States and many other areas of the world today, blood transfused is cross matched only for the ABO system and the Rh antigen. Prophylaxis is not available to prevent these cases. The fetal RhD antigen status can be determined by cordocentesis, DNA-based Rh typing using amniotic fluid, or chorionic villus sampling or by using fetal cell free DNA in maternal plasma [26].

\section{Infection}

There are several infections that may cause fetal anemia and hydrops. The most common cause in these cases is represented by Parvovirus B19 infection [27].The mechanism of fetal anemia in cases of parvovirus infection is secondary to the fetal erythroid progenitor cells infection with shortened half-life of erythrocytes, causing anemia, high output cardiac failure, and non-immune hydropsfetalis (NIHF). Syphilis, toxoplasmosis, coxackie virus, herpes simplex virus, rubella and CMV have been implicated as rare causes of fetal anemia and hydrops. The reason for fetal anemia in these conditions is less clear.

\section{Twin gestations (TAPS)}

Fetal anemia can occur in monochorionic twin gestations complicated by twin anemia-polycythemia sequence (TAPS) [28]. The diagnosis of TAPS has been reported using the MCA-PSV $>1.5$ Mom in the donor twin and MCA-PSV $<0.8$ MoM in the recipient twin. Fetal transfusion has been reported successfully in these cases.

\section{Diagnosis of Fetal anemia}

The diagnosis of fetal anemia includes invasive and non-invasive methods.

\section{Invasive methods}

The diagnosis of fetal anemia is achieved indirectly by amniocentesis (for many cases of red cell alloimmunization that cause hemolysis) with the bilirubin levels measured in the amniotic fluid or directly by cordocentesis for all cases of fetal anemia. The risk of cordocentesis is reported to be $1 \%$ [29].

\section{Noninvasive methods}

The blood velocity is increased in the severely anemic fetus due to a lower blood viscosity and to a higher cardiac output found in anemic fetuses [25]. Doppler ultrasonography of the middle cerebral artery peak systolic velocity (MCA PSV) is the parameter of choice to detect fetal anemia, because it is easy to get an angle of zero degrees between the ultrasound beam and the direction of blood flow at the MCA.

The MCA PSV 1.5 MoM is used to differentiate between anemic and non-anemic fetuses (Figure 1). The sensitivity of a single value of MCA-PSV in fetuses at risk for anemia is $100 \%$ (CI: $0.86-1.0)$ with a false positive rate of $12 \%$. However, the use of the MCA-PSV trend decreases the false positive rate to less that $5 \%$. The MCA-PSV can be used to accurately predict anemia in all cases of fetal anemia [30,31]. It has been reported

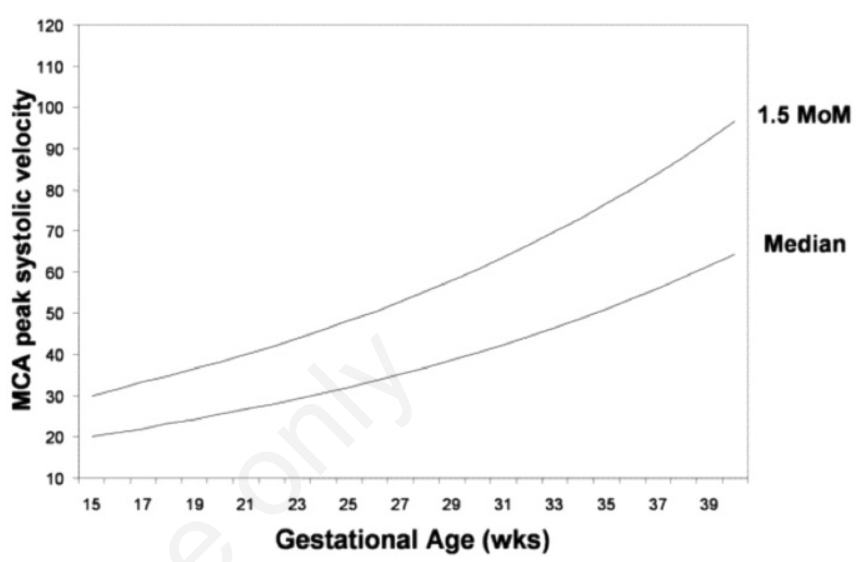

Figure 1. Middle cerebral artery peak systolic velocity multiples of the median with advancing gestation. A MCA-PSV 1.5 MoM is the cut-off used to differentiate between anemic cases (value above 1.5 MoM) and non-anemic cases. From Mari G et al. N Engl J Med 2000; 342:9-14.
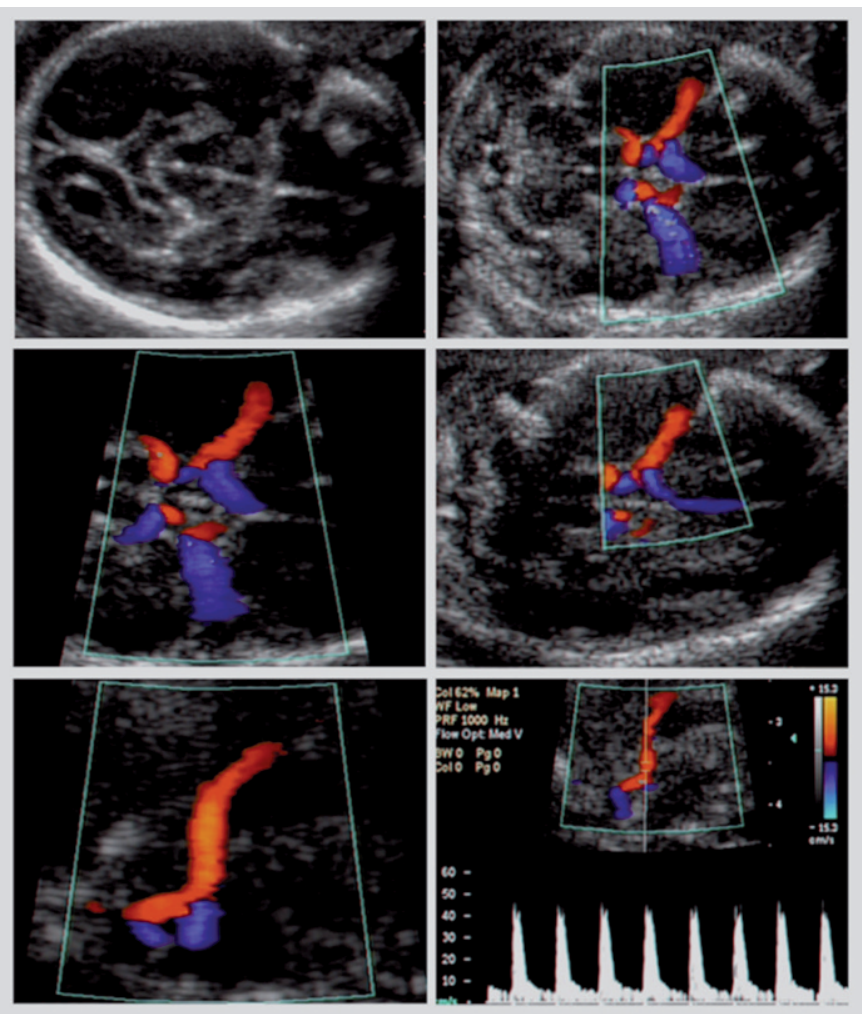

Figure 2. Steps for the correct assessment of the middle cerebral artery peak systolic velocity. From Mari G, et al. J Ultrasound Med 2005; 24:425-30. 
that the MCA-PSV is the best test to determine if a fetus is anemic because it has a higher sensitivity, specificity, positive and negative predictive value than the delta OD450 [32]. The steps for correct measurement of the MCA-PSV are reported in Figure 2[33]. Retrospective studies has suggested that the MCA-PSV could be used even in previously transfused fetuses [34].

\section{Prevention}

\section{Red cell alloimmunization}

Anti-D immune globulin prophylaxis, properly given, prevents $>99 \%$ of cases of $\mathrm{Rh}(\mathrm{D})$ alloimmunization if given both antepartum and postpartum. It should be given to all $\mathrm{Rh}(\mathrm{D})$ negative women with a negative antibody screen at 28 weeks, and if the neonate is $\mathrm{Rh}(\mathrm{D})$ positive, within $\mathbf{7 2}$ hours after birth. The optimal dose to administer, however, is unknown. Anti-D immunoglobulin prophylaxis is $300 \mathrm{mcg}$ ( 1 mcg $=5 \mathrm{IU}$ ) at 28 weeks, as well as after delivery if the neonate is $\mathrm{Rh}(\mathrm{D})$ positive. In the United Kingdom a 100 mcg dose administered at 28 and 34 weeks is also used. Anti-D immune globulin 300 micrograms protects against $30 \mathrm{~mL}$ of fetal whole blood or $15 \mathrm{~mL}$ of fetal red blood cells in the maternal circulation. In special circumstances (abdominal trauma, abruption, manual extraction of the placenta, etc.) a Kleihauer-Betke (KB) test should be done to determine the number of fetal cells that has entered the maternal circulation, and hence the appropriate dose of anti-D immune.

Currently, there is no prophylactic immune globulin to prevent Kell alloimmunization/other red cell antigens.

\section{FETAL GROWTH}

Obstetrics depends on gestational age (GA) and fetal weight (FW). Accurate ultrasound examination performed before 20 weeks of gestation enables true GA to be estimated. On the other hand, estimation of FW (EFW) using standard biometric parameters, usually related to geometric dimensions of the fetal head, abdomen and long bones of extremities, is still problematical [35]. Monitoring of fetal growth is fundamental in modern perinatology, because it is strictly related to fetal/neonatal wellbeing [36-39].

In the last 30 years, methods have been developed to improve EFW accuracy, most based on formulae derived by regression analysis, or on physical models. Artificial neural networks (ANNs) and volumetric methods based on three-dimensional (3D) ultrasonography were also recently proposed $[40,41]$. Although attempts to reduce statistical sample errors and lack of generalization power by selecting the most accurate and representative models have been made, a percentage mean absolute error (MAE) less than $7-8 \%$ of the true BW has never been achieved in current clinical practice, with $25 \%$ (or more) of estimates having an absolute error (AE) over $10 \%$.

Clinicians distinguish these two critical intervals of weight from an intermediate one, that typically ranges from 2,500 to 4,000 g. Almost all models for EFW exhibit a worsening of accuracy in critical weight classes (below 2,500 g and above 4,000 g) where lower/higher weights are usually over/under-estimated $[42,43]$. Most mathematical models are derived from statistical regressions and account nonlinearly for ultrasound measurements by fitting experimental data. They are therefore most accurate for intermediate weights, where experimental data has higher density, and produce increasing biases going from median to lower or higher FWs where data density progressively decreases.

The use of mathematical models specialized for specific FW and/or GA ranges can therefore be dangerous and not significantly better than those applicable to the entire fetal population. All other efforts to decrease $\mathrm{AE} \%$ by introducing correction factors in the algorithms and new information, such as amniotic fluid volume, number of fetuses and maternal pathologies, or non-routine echobiometric parameters, have failed to bring effective improvements. Specifically, three-dimensional (3D) ultrasound enables volumetric parameters such as fetal thigh, upper arm and abdomen to be measured for EFW. Although preliminary studies seems to indicate improvements, doubts remain about the utility of 3D for a substantial improvement in the accuracy of EFW [44]. Moreover, 3D ultrasound systems are expensive, not as widespread as 2D systems, and unfamiliar for operators doing routine fetal biometry.

Today, about ten models are considered to give the best, not significantly different performances and none give a MAE\% below 7-8\%.

The Fetal Weight Index (FWI) [45], a new informative probability model to improve fetal weight estimation accuracy (Figure 3), gives to operators:

a) the most reliable fetal weight estimation, reached evaluating simultaneously all fetal biometry parameters (biparietal diameter - BPD, head circumference - HC, abdominal circumference - AC, femur length - FL);

b) the most reliable weight related to each biometric parameter;

c) the probability of accuracy of each parameter in respect to others, expressed in percentage.

On the base of the estimated weight obtained by introducing in the system the fetal biometric measurements detected by operators, FWI gives the percentage of accuracy (correctness) of the estimation itself and, at the same time, gives the percentage of accuracy of each biometric parameter detected, thus allowing the reevaluation and the possible correction of data that seem less congruent with the estimation.

In clinical practice, independently from which formula is used, several intrinsic human and instrumental errors in the measurement procedure affect the accuracy 
of the fetal weight estimation, so that a mean absolute percentage error less than $7-8 \%$ has never been achieved with the $25 \%$ (or more) of estimates having a mean absolute percentage error even greater than $10 \%$. Unfortunately, this error is still too high to consider the method fully reliable for clinical decision-making. Moreover, the error arises when the estimation is focalized on the prenatal identification of macrosomic or intrauterine growth restricted fetuses; in fact, in these conditions, the estimation error easily exceeds $10-15 \%$. Using the FWI it is possible to highlight when the estimate obtained has a low percentage of accuracy. The reason can be related to 3 different situations: a particular biological condition of that specific fetus (biological variability), a wrong biometry detection (human error), or both. In particular, the percentage of accuracy of each fetal biometric parameter measured gives real time information about its congruity with the others: low percentages of accuracy means that the fetus could be wrongly measured or, correctly measured, but biologically different from others. In conclusion, all the information obtained by FWI allow a rational interaction between operator and software, reducing human error and enhancing fetal weight estimation accuracy.

\section{CERVICAL INSUFFICIENCY}

The term "cervical insufficiency" was used to describe a disorder in which painless cervical dilation led to recurrent second trimester pregnancy losses/births of otherwise normal pregnancies. As this necessitates a poor pregnancy outcome before making the diagnosis, and as we have modern ways to instead detect this condition, better modern means of making the diagnosis should be used, based on recent evidence.

\section{Diagnosis}

The diagnosis of cervical insufficiency is preferably made by a combination of historic factors and transvaginal ultrasound (TVU) measurement of cervical length (CL).

- TVU cervical length $<25 \mathrm{~mm}$ and/or advanced cervical changes on physical examination before 24 weeks of gestation in women with either:

- One or more prior pregnancy losses or preterm births at 14 to 36 weeks, and/or
BPD

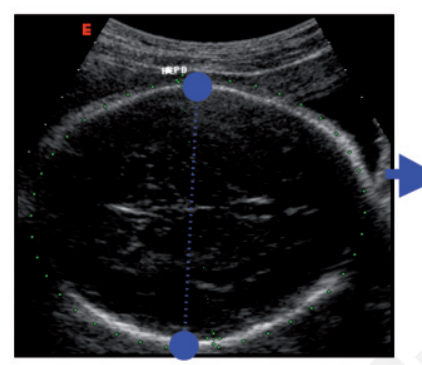

HC

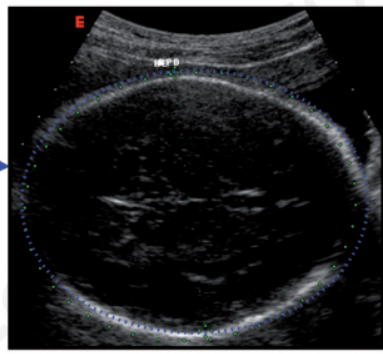

AC

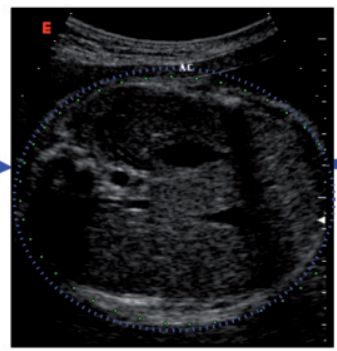

FL

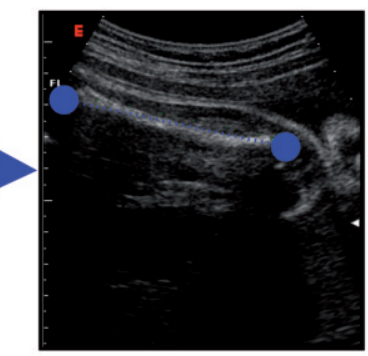

\section{Biparietal diameter \\ Head circumference}

Abdominal circumference

Femur length

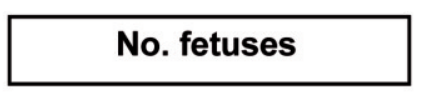

Amniotic fluid

\section{Gestational age}

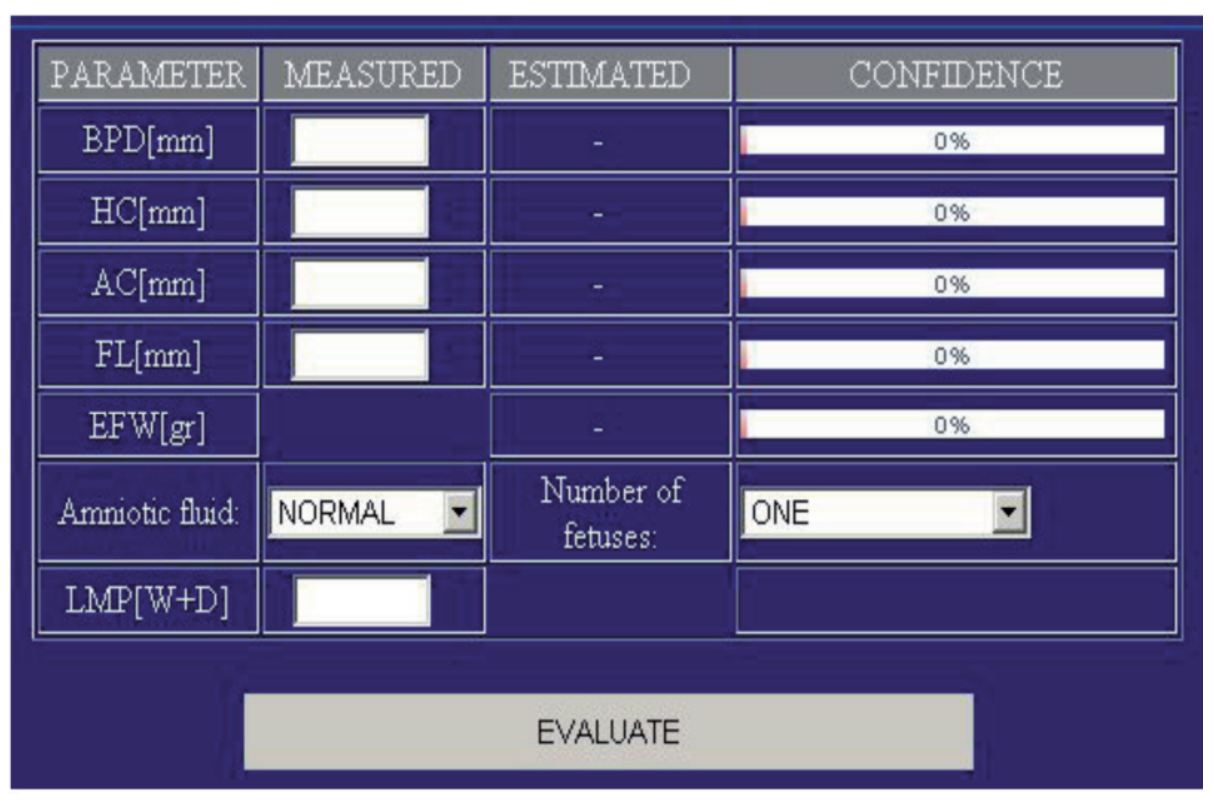

Figure 3. Fetal weight Index, Graphic user-interface of interactive software for fetal echobiometry control and correction. 
- Other significant risk factors for cervical insufficiency.

Risk factors for cervical insufficiency can be acquired or congenital; acquired factors are more common. Acquired factors are obstetric trauma (e.g. cervical laceration); mechanical dilation (e.g. dilation and curettage $[D \& C]$, dilation and evacuation [D\&E], pregnancy termination, hysteroscopy); and even reatment of cervical intraepithelial neoplasia. Congenital factors are collagen abnormalities (e.g., Ehlers-Danlos syndrome); uterine anomalies; Diethylstilbestrol (DES) exposure; and biologic variation.

Using historic factors alone, cervical insufficiency is defined as painless cervical dilatation leading to recurrent second trimester pregnancy losses/births. This definition precludes diagnosis of cervical insufficiency until at least two pregnancy losses/births before $\mathbf{2 8}$ weeks of gestation have occurred.

The diagnosis of cervical insufficiency is usually limited to singleton gestations because the pathogenesis of delivery at 14 to 28 weeks in multiple gestations is usually unrelated to a weakened cervix. In addition, preterm labor, infection, abruptio placenta, and bleeding placenta previa should be excluded. The diagnosis of cervical insufficiency cannot be made or excluded outside of pregnancy. Evaluation of cervical function with dilators, balloons, or hysteroscopy is not helpful. Ultrasound, magnetic resonance imaging, or hysterosalpingography may reveal a uterine anomaly, which is a risk factor for cervical insufficiency, but is not diagnostic.

\section{Management \\ History-indicated cerclage}

History-indicated cerclage at 12 to 14 weeks is indicated for women who meet all of the following criteria [47]:

- Two or more consecutive prior second trimester pregnancy losses or three or more early ( $<34$ weeks) preterm births.

We also treat these women with 17-alpha-hydroxyprogesterone caproate weekly from 16 to 36 weeks of gestation [47].

\section{Ultrasound-indicated cerclage}

In the vast majority of women with prior PTB, we usually initiate TVU cervical length screening at 16weeks, and at the same gestational age start to administer 17-alpha-hydroxyprogesterone caproate prophylaxis. We perform a cerclage only if cervical length decreases to $<25 \mathrm{~mm}$ before 24 weeks [48].

In randomized trials, progesterone prophylaxis with 17 alpha hydroxy-progesterone caproate starting at 16 to 20 weeks in women with a history of spontaneous preterm birth and continuing until 36 weeks reduced the risk of recurrent preterm birth [47]. Placement of cerclage upon identification of a short cervix ("ultrasound-indicated cerclage") is effective in reducing preterm birth [49], results in pregnancy outcomes com- parable to those with history-indicated cerclage [48], and avoids cerclage in about 60 percent of patients with a suggestive history [50].

In a meta-analysis of randomized trials of women with singleton gestation and prior spontaneous preterm birth and short cervical length $<25 \mathrm{~mm}$ before 24 weeks, treatment with ultrasound-indicated cerclage significantly lowered total neonatal morbidity and mortality ( 15.6 versus 24.8 percent without cerclage; RR 0.64, 95\% CI 0.45-0.91), presumably because cerclage significantly reduced the frequency of preterm birth (delivery <35 weeks RR $0.70,95 \%$ CI $0.55-0.89 ; 28.4$ percent versus 41.3 percent in women without cerclage) [49].

In another meta-analysis of randomized trials of women with singleton gestations and prior preterm birth managed either by (1) cervical length screening with cerclage for short cervical length or (2) history-indicated cerclage, patients with ultrasound-indicated versus history-indicated cerclage had similar rates of preterm birth before 37 weeks ( 31 versus 32 percent, RR $0.97,95 \%$ CI $0.73-1.29$ ), preterm birth before 34 weeks (17 versus 23 percent, RR $0.76,95 \%$ CI $0.48-1.20$ ), and perinatal mortality ( 5 versus 3 percent, RR 1.77, 95\% CI $0.58-5.35)$, and only 42 percent developed a short cervical length and received cerclage [50].

Usually, cervical length screening initiates at 16 weeks, but may screen as early as 12 weeks in women with early second trimester losses, recurrent second trimester losses, or prior large cold knife conization [51]. In women with prior preterm birth at 28 to 36 weeks, we initiate screening at 16 weeks. Ultrasound examination is generally repeated every two weeks until 24 weeks as long as the cervical length is $\geq 30 \mathrm{~mm}$, and increased to weekly if cervical length is 25 to 29 $\mathrm{mm}$, with the expectation that preterm cervical changes will precede overt preterm labor or membrane rupture symptoms by three to six weeks [52]. Transvaginal ultrasound screening is usually discontinued at 24 weeks of gestation, as cerclage is not usually performed after this time.

Intramuscular progesterone supplementation continues until 36 weeks, whether or not a cerclage is placed. One post-hoc analysis of data from a randomized trial observed that the rate of early preterm birth trended lower in women who received both 17-alpha-hydroxyprogesterone caproate and ultrasound-indicated cerclage than in those who received either therapy alone (preterm birth <28 weeks: both interventions: 9 percent versus cerclage alone: 17 percent and progesterone alone: 15 percent; preterm birth $<32$ weeks: both interventions: 17 percent versus cerclage alone: 25 and progesterone alone: 21 percent) [53].

\section{Physical exam-indicated cerclage}

Rarely, a woman presents before 24 weeks with minimal or no symptoms and physical examination reveals a dilated cervix. Placement of a physical exam-indi- 
cated cerclage when a dilated cervix and visible membranes are detected on digital examination at $<24$ weeks is associated with prolongation of pregnancy and improved pregnancy outcome compared to expectant management in a small randomized trial, and retrospective cohort studies [54,55].

\section{CONCLUSIONS}

The development of Maternal-Fetal Medicine is contributing to an incredible improvement of maternal well-being and of neonatal health. Even though there is an increasing trend in the number of pregnancy at risk, the development of maternal-fetal technologies is contributing to a good outcome.

The combination of new biophysical and biochemical markers is enriching this area of medicine, in which one patient (fetus) is not able to answer to any question. Therefore, it is mandatory to do more research investigating resources in order to take care of the maternalfetal unit and to allow good outcome of pregnancy.

\section{REFERENCES}

1. Wellesley D, Dolk H, Boyd PA, et al. Rare chromosome abnormalities, prevalence and prenatal diagnosis rates from populationbased congenital anomaly registers in Europe. Eur J Hum Genet. 2012;20:521-6.

2. Wenstrom KD. Evaluation of Down syndrome screening strategies. Semin Perinatol. 2005;29:219-24.

3. Aagaard-Tillery KM, Malone FD, Nyberg DA, et al. Role of second-trimester genetic sonography after Down syndrome screening. Obstet Gynecol. 2009;114:1189-96.

4. Chiu RW, Chan KC, Gao Y, et al. Noninvasive prenatal diagnosis of fetal chromosomal aneuploidy by massively parallel genomic sequencing of DNA in maternal plasma. Proc Natl Acad Sci USA 2008;105:20458-63.

5. Palomaki GE, Kloza EM, Lambert-Messerlian GM, et al. DNA sequencing of maternal plasma to detect Down syndrome: an international clinical validation study. Genet Med 2011;13:913-20.

6. Bianchi DW, Platt LD, Goldberg JD, et al. Genome-wide fetal aneuploidy detection by maternal plasma DNA sequencing. Obstet Gynecol 2012;119;890-901.

7. Norton ME, Brar H, Weiss J, et al. Non-Invasive Chromosomal Evaluation (NICE) Study: results of a multicenter prospective cohort study for detection of fetal trisomy 21 and trisomy 18. Am J Obstet Gynecol 2012;207:137.e1-8.

8. Guedj F, Bianchi DW. Noninvasive prenatal testing creates an opportunity for antenatal treatment of Down syndrome. Prenat Diagn 2013;33:614-8.

9. Jiang J, Jing $Y$, Cost GJ, et al. Translating dosage compensation to trisomy 21. Nature 2013;500:296-300.

10. Fan $H C, G u W$, Wang J, et al. Non-invasive prenatal measurement of the fetal genome. Nature 2012;487:320-4.

11. Srinivasan A, Bianchi DW, Huang $H$, et al. Noninvasive detection of fetal subchromosome abnormalities via deep sequencing of maternal plasma. Am J Hum Genet 2013;92:167-76.

12. Lazarin GA, Haque IS, Nazareth $S$, et al. An empiracal estimate of carrier frequencies for 400+ causal Mendelian variants: results from an ethnically diverse clinical sample of 23,453 individuals. Genet Med 2013;15:178-86.

13. Wapner RJ, Martin CL, Levy B, et al. Chromosomal microarray versus karyotyping for prenatal diagnosis. $N$ Engl $J$ Med 2012;367:2175-84.

14. Reddy UM, Page GP, Saade GR, et al. Karyotype versus microarray testing for genetic abnormalities after stillbirth. $N$ Engl J Med 2012;367:2185-93.

15. Barcroft J. Researches on prenatal life. Oxford: Blackwell Scientific Publication; 1946.

16. Barclay AE, Franklin KJ, Prichard MML. The foetal circulation and cardiovascular system, and changes that they undergo at birth. Oxford: Blackwell Scientific Publication; 1944.

17. Dawes GS. Foetal and neonatal physiology: a comparative study of changes at birth. Chicago: Year Book Medical Publisher; 1968.

18. Rudolph AM, Heymnn MA. The circulation of the fetus in utero. Methods for studying distribution of blood flow, cardiac output and organ blood flow. Circul Res 1967;21:163-84.

19. Fitzgerald DE Drumm JE. Non invasive measurements of fetal human circulation using ultrasound: a new method. Br Med J 1977;2:1450-1.

20. Schmidth KG, Di Tommaso M, Silverman NH, Rudolph AM. Doppler echocardiographic assessment of fetal descending aortic and umbilical blood flows. Validation studies in fetal lambs. Circulation 1991;83:1731-7.

21. Ferrazzi E, Bellotti M, Galan H, et al. Doppler investigation in intrauterine growth restriction--from qualitative indices to flow measurements: a review of the experience of a collaborative group. Ann N Y Acad Sci 2001;943:316-25.

22. Harman CR, Baschat AA. Comprehensive assessment of fetal wellbeing: which Doppler tests should be performed? Curr Opin Obstet Gynecol 2003;15:147-57.

23. Mari $G$, for the Collaborative Group for Doppler. Assessment of the blood velocity in anemic fetuses non invasive diagnosis by doppler ultrasonography of fetal anemia due to maternal red-cell alloimmunization. N Engl J Med 2000;342:9-14.

24. Baschat $A A$.Venous doppler evaluation of the growth-restricted fetus. Clin Perinatol 2011;38:103-12.

25. Mari G, Deter $R L$, Carpenter $R L$, et al. Noninvasive diagnosis by Doppler ultrasonography of fetal anemia due to maternal red-cell alloimmunization. Collaborative Group for Doppler Assessment of the Blood Velocity in Anemic Fetuses. New Engl J Med 2000;342:9-14.

26. Bombard AT, Akolekar R, Farkas DH, et al. Fetal RHD genotype detection from circulating cell-free fetal DNA in maternal plasma in non-sensitized RhD negative women. Prenat Diagn 2011;31:802-8.

27. Giorgio E, De Oronzo MA, Iozza I, et al. Parvovirus B19 during pregnancy: a review. J Prenat Med 2010;4:63-6.

28. Slaghekke F, Kist WJ, Oepkes D, et al. Twin anemia-polycythemia sequence: diagnostic criteria, classification, perinatal management and outcome. Fetal Diagn Ther 2010;27:181-90.

29. Ghidini A, Sepulveda W, Lockwood CJ, Romero R. Complications of fetal blood sampling. American journal of obstetrics and gynecology 1993;168:1339-44.

30. Delle Chiaie L, Buck G, Grab D, Terinde R. Prediction of fetal anemia with Doppler measurement of the middle cerebral artery peak systolic velocity in pregnancies complicated by maternal blood group alloimmunization or parvovirus B19 infection. Ultrasound Obstet Gynecol 2001;18:232-6.

31. Cosmi E, Mari G, Delle Chiaie L, et al. Noninvasive diagnosis by Doppler ultrasonography of fetal anemia resulting from parvovirus infection. Am J Obstet Gynecol 2002;187:1290-3.

32. Oepkes D, Seaward PG, Vandenbussche FP, et al. Doppler ultra- 
sonography versus amniocentesis to predict fetal anemia. N Engl $J$ Med 2006;355:156-64.

33. Mari G, Abuhamad AZ, Cosmi E, et al. Middle cerebral artery peak systolic velocity: technique and variability. J Ultrasound Med 2005;24:425-30.

34. Detti $L, O z U$, Guney I, et al. Doppler ultrasound velocimetry for timing the second intrauterine transfusion in fetuses with anemia from red cell alloimmunization. Am J Obstet Gynecol 2001;185:1048-51.

35. Barel O, Maymon R, Vaknin Z, et al. Sonographic fetal weight estimation - is there more to it than just fetal measurements? Prenat Diagn 2014;34:50-5.

36. Chauhan SP, Cole J, Sanderson M, et al, Suspicion of intrauterine growth restriction: use of abdominal circumference alone or estimated fetal weight below 10\%. J Matern Fetal Neonatal Med 2006;19:557-62.

37. Melamed N, Yogev Y, Meizner I, et al. Prediction of fetal macrosomia: effect of sonographic fetal weight-estimation model and threshold used. Ultrasound Obstet Gynecol 2011;38:74-81.

38. Severi $F M$, Bocchi $C$, Imperatore A, et al. Ultrasound estimated fetal weight slightly below the median is associated with increased risk of spontaneous preterm birth. Prenat Diagn 2012;32:588-91.

39. Severi FM, Bocchi $C$, Visentin A, et al. Uterine and fetal cerebral Doppler predict the outcome of third-trimester small-for-gestational age fetuses with normal umbilical artery Doppler. Ultrasound $\mathrm{Ob}$ stet Gynecol 2002;19:225-8.

40. Benavides-Serralde A, Hernandez-Andrade E, Fernandez-Lara A, et al. Accuracy of different equations for estimating fetal weight. Gynecol Obstet Invest 2011;72:264-8.

41. Chuang L, Hwang JY, Chang CH, et al. Ultrasound estimation of fetal weight with the use of computerized artificial neural network model. Ultrasound Med Biol 2002;28:991-6.

42. Lindell G, Källén K, Maršál K. Ultrasound weight estimation of large fetuses. Acta Obstet Gynecol Scand 2012;91:1218-25.

43. Thompson JL, Kuller JA, Rhee EH. Antenatal surveillance of fetal growth restriction. Obstet Gynecol Surv 2012;67:554-65.

44. Schild RL, Fimmers $R$, Hansmann M. Fetal weight estimation by three-dimensional ultrasound. Ultrasound Obstet Gynecol 2000;16:445-52.

45. Cevenini G, Severi FM, Bocchi $C$, et al. An informative probability model enhancing real time echobiometry to improve fetal weight estimation accuracy, Med Bio Eng Comput 2008;46:109-20.

46. MRC/RCOG Working Party on Cervical Cerclage. Final report of the Medical Research Council/Royal College of Obstetricians and Gynaecologists multicentre randomised trial of cervical cerclage. $\mathrm{Br}$ J Obstet Gynaecol 1993;100:516.

47. Meis PJ, Klebanoff $M$, Thom $E$, et al. Prevention of recurrent preterm delivery by 17 alpha-hydroxyprogesterone caproate. $N$ Engl J Med 2003;348:2379.

48. Society for Maternal-Fetal Medicine Publications Committee, with assistance of Vincenzo Berghella. Progesterone and preterm birth prevention: translating clinical trials data into clinical practice. Am J Obstet Gynecol 2012;206:376.

49. Berghella V, Rafael TJ, Szychowski JM, et al. Cerclage for short cervix on ultrasonography in women with singleton gestations and previous preterm birth: a meta-analysis. Obstet Gynecol 2011; 117:663.

50. Berghella V, Mackeen AD. Cervical length screening with ultrasound-indicated cerclage compared with history-indicated cerclage for prevention of preterm birth: a meta-analysis. Obstet Gynecol 2011;118:148.

51. Berghella $V$, Talucci $M$, Desai A. Does transvaginal sonographic measurement of cervical length before 14 weeks predict preterm delivery in high-risk pregnancies? Ultrasound Obstet Gynecol 2003; 21:140.

52. Iams JD, Cebrik D, Lynch C, et al. The rate of cervical change and the phenotype of spontaneous preterm birth. Am J Obstet Gynecol 2011;130:e1-6.

53. Berghella V, Figueroa D, Szychowski JM, et al. 17-alpha-hydroxyprogesterone caproate for the prevention of preterm birth in women with prior preterm birth and a short cervical length. Am J Obstet Gynecol 2010;202:e1-6.

54. Althuisius SM, Dekker GA, van Geijn HP, et al. Cervical incompetence prevention randomized cerclage trial (CIPRACT): study design and preliminary results. Am J Obstet Gynecol 2000;183:823-9.

55. Pereira L, Cotter A, Gómez $R$, et al. Expectant management compared with physical examination-indicated cerclage (EM-PEC) in selected women with a dilated cervix at 14(0/7)-25(6/7) weeks: results from the EM-PEC international cohort study. Am J Obstet Gynecol 2007;197:e1-8. 